\title{
Takayasu's Arteritis-A Case Report and a Brief Review of Diagnostic Imaging
}

\section{Amit Nandan D Dwivedi* and Siddiqui Z}

Department of Radiodiagnosis and Imaging, Banaras Hindu University, India

*Corresponding author: Amit Nandan Dhar Dwivedi, Department of Radiodiagnosis and Imaging, Institute of Medical Sciences, Banaras Hindu University, Varanasi, Uttar

Pradesh, India, Tel: +91-9793856930; Email: amitnandan21@yahoo.com

\section{Case Report}

Volume 3 Issue 3

Received Date: July 09, 2019

Published Date: July 23, 2019

DOI: $10.23880 /$ crij-16000150

\begin{abstract}
Takayasu's arteritis (TA) is a rare, idiopathic, chronic vasculitis, mainly involving the aorta and its major branches. Here, we present the case of an eighteen year old female who presented with the complaints of fever, palpitations and breathlessness. She was found to have bilateral carotid bruit and a significant discrepancy in blood pressure of bilateral upper limbs. She was later diagnosed as having Takayasu's arteritis on the basis of typical imaging features. In this case report, we tend to highlight the importance of imaging in TA along with its typical imaging features and advantages \& limitations of various imaging modalities.
\end{abstract}

Keywords: Aorta; Limb; Stenosis; Palpitations; Luminal Stenosis; Arthralgia

Abbreviations: TA: Takayasu's Arteritis; CTA: CT Angiography; MRI: Magnetic Resonance Imaging; ACR: American College of Rheumatology.

\section{Introduction}

Takayasu's arteritis (TA), also known as 'Pulseless disease' or 'Aortoarteritis', is a form of granulomatous arteritis which affects large and medium sized arteries, primarily the aorta and its major branches [1]. The etiology of Takayasu's arteritis remains largely unknown. The primary pathologic process is inflammatory, with several etiologic factors being proposed in the past, including infections and circulating antibodies due to an immune mediated process [2]. It is most frequently seen in young women. Early reports suggested that the disease was confined to females from Eastern Asia, but it has now been recognised worldwide in both sexes, although disease manifestations vary between populations.
Inflammation of the vessel wall leads to wall thickening, stenosis, occlusion, dilatation, and aneurysmal formation in the affected vessels. Diminished or absent pulses are present in $84-96 \%$ of patients, associated with limb claudication and blood pressure discrepancies between the two arms [1]. The symptoms are secondary to the involved artery, and it may evolve into a life-threatening condition. Timely diagnosis is crucial to prevent permanent end organ damage.

\section{Case Report}

An eighteen year old female was admitted to the medicine department with the chief complaints of on and off fever for last four years and palpitations \& breathlessness for the last six months. A history of weight loss was also present. There was no history of chest pain, claudication, syncope or blurring of vision. On general examination, patient was conscious, cooperative and 


\section{Clinical Radiology \& Imaging Journal}

oriented to time, place and person. Bilateral brachial and radial arteries showed feeble pulses and a significant difference in blood pressure were recorded in bilateral upper limbs. Jugular venous pressure was raised and bilateral carotid bruits were present. Cardiac examination revealed a systolic murmur over pulmonary area, loud P2 and apex palpable in sixth intercostal space, lateral to midclavicular line.

The patient was referred to the radiology department with an aim of evaluating the cause for carotid bruits and the discrepancy in blood pressure of bilateral upper limbs. A colour doppler study of neck vessels, revealed diffuse circumferential thickening involving bilateral common carotid arteries along with luminal narrowing (Figures 1 \& 2) and significant velocity changes. The doppler study of bilateral upper limbs was unremarkable. Hence, the patient was advised CT Angiography (CTA) for further evaluation which revealed concentric mural thickening involving the ascending aorta, aortic arch, descending thoracic (Figures $3 \& 4$ ) and abdominal aorta. No associated calcification was seen. Bilateral common carotid arteries showed circumferential mural thickening causing significant luminal stenosis (Figure 5), as was seen on colour doppler study. Bilateral subclavian arteries were also involved. Proximal part of superior mesenteric artery showed concentric wall thickening with associated luminal narrowing; however, the celiac axis \& inferior mesenteric arteries were spared and showed normal calibre \& wall thickness. Long segment stenotic lesion was noted in right renal artery, near its origin; left renal artery was spared. No obvious evidence of any ectasia or aneurysm was noted in the involved vessels. No signs of any end organ damage or ischemia were noted. Associated left ventricular hypertrophy and cardiomegaly was seen. Pulmonary and coronary arteries were not involved. On the basis of clinical and CTA features, a diagnosis of Takayasu's arteritis was made.

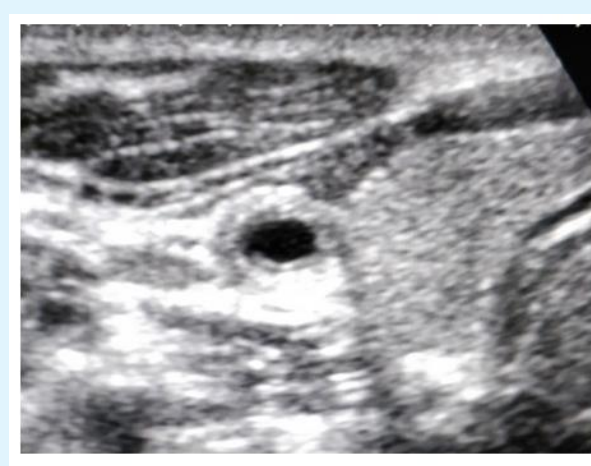

1

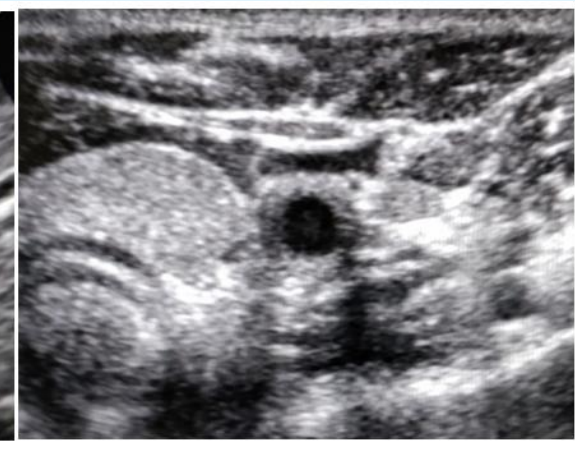

2

Figures 1 \& 2: Gray scale sonographic images showing bilateral common carotid arteries. Diffuse, homogenous, circumferential mural thickening of bilateral common carotid arteries is noted along with luminal narrowing.

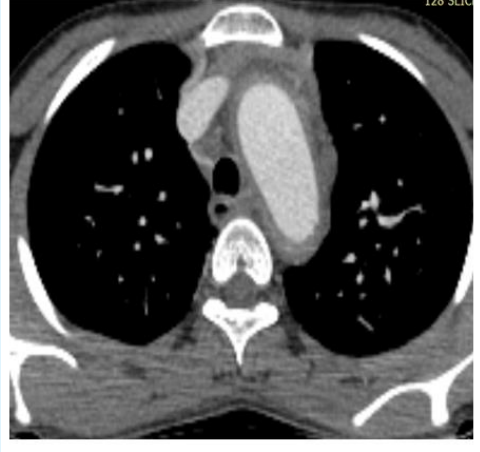

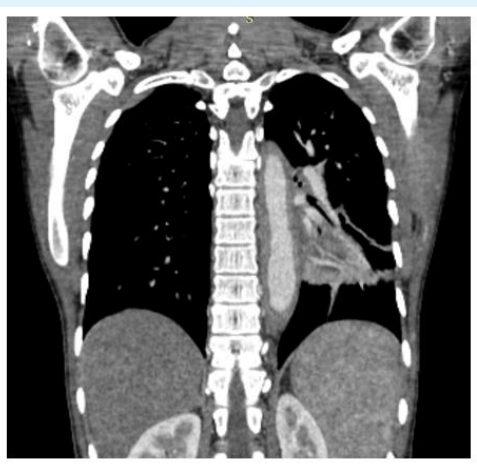

4

Figures 3 \& 4: Axial and coronal post contrast CTA images showing diffuse, concentric mural thickening involving the aortic arch and the descending thoracic aorta. 


\section{Clinical Radiology \& Imaging Journal}

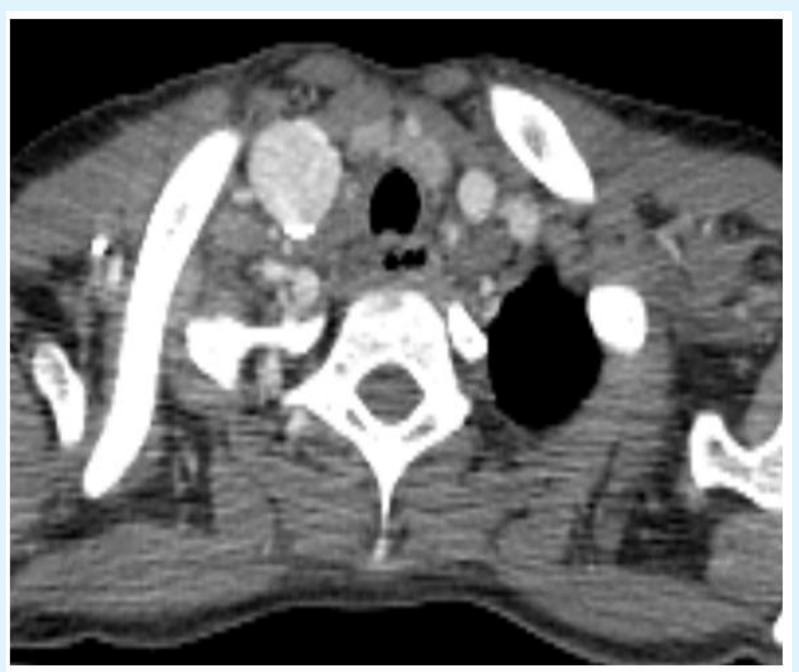

Figure 5: Axial post contrast CT angiography (CTA) image of the same patient showing mural thickening with significant luminal stenosis involving the bilateral common carotid arteries.

\section{Discussion}

Takayasu's arteritis (TA), also known as 'Pulseless disease' or 'Aortoarteritis', is a rare and chronic idiopathic inflammatory condition which is most frequently observed in young women, with females being affected eight times more than men [3]. The female to male ratio appears to decline from Eastern Asia towards the West [1]. The median age of onset is 25 years; however, approximately $25 \%$ cases have an onset before the age of 20 and $10-20 \%$ present after the age of 40 . It is more common in Asia but is not racially or geographically restricted [4].

Clinical manifestations of TA are non-specific and can be divided into two categories: (1) Constitutional symptoms, which include fever, weight loss, malaise, arthralgias \& headache and (2) Symptoms due to vascular insufficiency, depending upon the vessel being involved. For example: Arterial hypertension and renal failure due to the involvement of renal arteries [5].

Indeed, imaging plays a crucial role in the diagnosis of TA. Sonography can be used for the initial evaluation of patients with suspected TA. The involved vessels show homogenous, circumferential mural thickening with associated narrowing. Vascular occlusions, aneurysms and velocity changes can be evaluated using doppler study. However, sonography cannot be used for the complete evaluation of aorta and its abdominal branches due to obscuration by bowel gas, nor is it of any use for the evaluation of thoracic aorta, coronary and pulmonary arteries.

Conventional angiography has been considered the 'gold standard technique' for diagnosis, but it is invasive. It often shows long segment, smooth tapering stenosis or frank occlusions and offers the added advantage of performing guided interventions in the same sitting. It can also be used for the measurement of central arterial pressure. However, it can be difficult to perform it in severe stenosis \& heavy calcification and it does not provide any information about the wall architecture. Hence, it cannot be used to differentiate between stenosis due to acute mural inflammation and stenosis due to chronic mural fibrosis [6].

Multidetector CT angiography (CTA) has emerged as a reliable non-invasive tool in the evaluation of patients with TA and has largely replaced conventional angiography for diagnostic purposes. The two most important CT angiography features in TA include mural thickening and luminal changes in the involved vessels. Mural thickening may be the most important finding in the early phases of the disease [1]. Calcification in the thickened wall has been observed in $27 \%$ of patients and it is usually transmural [7]. On the post-enhanced CTA images, a double ring enhancement pattern of the thickened wall has been described, which is best seen in the venous phase $[7,8]$. A faint inner ring and an obviously visible outer ring are usually observed. Most commonly seen finding associated with mural thickening is, luminal stenosis and the stenotic lesions are most frequently observed in the subclavian and common carotid arteries, followed by the renal arteries. Complete occlusion, ectasia and aneurysm of the vessels can also be seen, but are less common $[9,10]$. Pulmonary and coronary artery involvement can be seen in $63.3 \%$ and $44.4 \%$ of patients, respectively [11]. CTA also helps is the evaluation of end organ status, such as signs of ischemia in brain and bowel, which can help in prognostication of these patients and in dictating the line of further management.

MRI is ideal for serial evaluation of TA patients due to its lack of need for ionizing radiation. Patients of TA are most frequently young females and hence, MRI is desirable to avoid excessive radiation exposure. MRI can be useful for early diagnosis of TA because of its ability to detect early wall changes and increase in thickness. Techniques like cine MRI can also detect hemodynamic changes such as aortic regurgitation (AR).Disadvantages of MRI include longer scan times, difficulty in visualizing 


\section{Clinical Radiology \& Imaging Journal}

small branch vessels and poor visualisation of calcifications.

MR Angiography provides detailed vascular information, including location, degree \& extent of stenosis and dilatation and patency of collateral vessels and grafts. However, its limitations include the possibility that vascular branch points maybe misinterpreted as occlusions and MIP images may falsely accentuate the degree of vascular stenosis [12].

Diagnosis of TA is based on typical clinical findings and imaging features. Several criterias, such as American College of Rheumatology (ACR) criteria or Sharma criteria can be used for the diagnosis $[13,14]$. Sharma criteria are basically a modification of the original Ishikawa criteria, and it is widely used. Treatment of TA consists of medical and surgical therapy. Corticosteroids are the cornerstone for the treatment of active TA. Surgical therapy and image guided interventions are used to treat stenotic lesions and aneurysms, especially involving the carotid, coronary and renal arteries.

This case report aims to highlight the importance of imaging modalities in the diagnosis, as well as, prognostication of patients with TA. A high degree of clinical suspicion alongwith appropriate imaging techniques is required for an accurate diagnosis, especially in patients presenting with atypical and non specific clinical features. The described imaging features, site \& extent of the vessels involved and signs of end organ ischemia, not only help in the diagnosis of TA, but are also helpful in deciding and prioritizing the further line of management for such patients, especially with respect to interventions and surgery.

\section{References}

1. Johnston SL, Lock RJ, Gompels MM (2002) Takayasu arteritis: a review. J ClinPathol 55(7): 481-486.

2. Espinoza JL, Ai S, Matsumura I (2018) New Insights on the Pathogenesis of Takayasu Arteritis: Revisiting the Microbial Theory. Pathogens 7(3): 61-67.

3. Mason JC (2010) Takayasu arteritis-advances in diagnosis and management. Nat Rev Rheumatol 6(7): 406-415.

4. Brunner J, Feldman BM, Tyrrell PN, KuemmerleDeschner JB, Zimmerhackl LB, et al. (2010) Takayasu arteritis inchildren and adolescents. Rheumatology 49(10): 1806-1814.
5. Park MC, Lee SW, Park YB, Chung NS, Lee SK (2005) Clinical characteristics and outcomes of Takayasu's arteritis: analysis of 108 patients using standardized criteria for diagnosis, activity assessment, and angiographic classification. Scand J Rheumatol 34(4): 284-292.

6. Blockmans D, Bley T, Schmidt W (2009) Imaging for large-vessel vasculitis. Curr Opin Rheumatol 21(1): 19-28.

7. Khandelwal N, Kalra N, Garg MK, Kang M, Lal A, et al. (2011) Multidetector CT angiography in Takayasu arteritis. Eur J Radiol 77(2): 369-374.

8. Matsunaga N, Hayashi K, Sakamoto I, Ogawa Y, Matsumoto $T$ (1997) Takayasu arteritis: protean radiologic manifestations and diagnosis. Radiographics 17(3): 579-594.

9. Lee GY, Jang SY, Ko SM, Kim EK, Lee SH, et al. (2012) Cardiovascular manifestations of Takayasu arteritis and their relationship to the disease activity: analysis of 204 Korean patients at a single center. Int J Cardiol 159(1): 14-20.

10. Soto ME, Melendez-Ramirez G, Kimura-Hayama E, Meave-Gonzalez A, Achenbach S, et al. (2011) Coronary CT angiography in Takayasu arteritis. JACC Cardiovasc Imaging 4(9): 958-966.

11. Cong XL, Dai SM, Feng X, Wang ZW, Lu QS, et al. (2010) Takayasu's arteritis: clinical features and outcomes of 125 patients in China. Clin Rheumatol 29(9): 973-981.

12. Andrews J, Al-Nahhas A, Pennell DJ, Hossain MS, Davies KA, et al (2004) Non-invasive imaging in the diagnosis and management of Takayasu's arteritis. Ann Rheum Dis 63(8): 995-1000.

13. Arend WP, Michel BA, Bloch DA, Hunder GG, Calabrese LH, et al. (1990) The American College of Rheumatology 1990 criteria for the classification of Takayasu arteritis. Arthritis Rheum 33(8): 11291134.

14. Sharma BK, Jain S, Suri S, Numano F (1996) Diagnostic criteria for Takayasu arteritis. Int J Cardiol 54: 141-147. 